\title{
Considering the Role of Experience in the Formation of Behavioral Biases from a Developmental, Cross-Cultural, and Evolutionary Perspective
}

\author{
Rebecca Williamson $^{1}$, Bethany MacDonald ${ }^{1}$, and Sarah F. Brosnan ${ }^{1,2^{*}}$ \\ ${ }^{\mathbf{1}}$ Department of Psychology, Georgia State University \\ ${ }^{\mathbf{2}}$ Neuroscience Institute, Center for Behavioral Neuroscience, and Language Research Center, Georgia State University \\ *Corresponding author (Email: Sarah.Brosnan@gmail.com)
}

Citation - Williamson, R., MacDonald, B., \& Brosnan, S.F. (2019). Considering the role of experience in the formation of behavioral biases from a developmental, cross-cultural, and evolutionary perspective. Animal Behavior and Cognition, 6(3), 179-193. https://doi.org/10.26451/abc.06.03.03.2019

\begin{abstract}
Humans make countless choices every day that affect their health, safety, and finances. Despite the high stakes, decision-making is often irrational from the viewpoint of traditional economics, i.e., the choices made are contrary to existing preferences. This leads to negative consequences for individuals and to inefficiencies in the exchange of goods. The fields of behavioral and experimental economics have made great strides in understanding these sub-optimal patterns of behavior, but we still cannot always predict human decisions. In this paper, we present developmental, cross-cultural, and comparative findings for three irrational tendencies (framing effects, the endowment effect, and inequity aversion) to illustrate two different patterns that experience can play in their formation. This analysis allows us to consider why these tendencies have emerged, what benefits they may bring to the decision-maker, and to propose types of interventions that may combat these tendencies. Throughout, we suggest where this approach of combining comparative and developmental work can address theoretical debates and practical questions within the field of behavioral economics.
\end{abstract}

Keywords - Decision making, Behavioral biases, Cognitive and perceptual development, Evolutionary theory, Information, Knowledge and uncertainty

Imagine your sister is getting married and you need to buy a gift. She has a new stand mixer on her registry. It is expensive, but this is your sister's wedding and you want to splurge for the occasion. The big question is: How will you pay for it? Do you take a deep breath and pull the money from that savings account you have painstakingly built? Or do you put it on your credit card and pay it (and the interest) off over a few months? According to traditional economic theory, people act rationally to maximize their existing preferences (Becker, 1976). Thus, this choice should be easy to make; assuming you value money, you should pay with your savings account to save the cost of interest charges. However, behavioral and experimental economists have shown that real-world behavior is not so cut and dry; sometimes people decide to do the opposite, either to save the pain of a hefty withdrawal by using their credit card instead of laying out their own money upfront, or avoid the risk of failing to re-fill their savings account by putting it on a credit card, where the interest will guarantee motivation to repay. A variety of external factors impact decision making, including how the information is presented, one's relation to the resources involved, and the social context in which a decision is situated. At times, this leads to apparently irrational choices - that is, decisions that go against preferences that were expressed in 
a different context (this is the definition of irrational decision making that we use throughout). An open question is why this is the case.

The focus of this review is to consider the role of experience in explaining why people act irrationally on decision-making tasks. Most behavioral data on economic decision making comes from research with (mostly Western) adults. Such work has been fundamental for discovering irrational tendencies and charting their scope and consequences. However, to predict how individuals will act across a wide variety of contexts, a better understanding of the mechanisms underlying decision making is needed. Much as it has enhanced our understanding of topics such as culture and cognitive ability, studying decision making from both developmental and comparative perspectives can provide valuable insights into these behaviors. Data from children and non-human primates, not to mention cross-cultural studies (Apicella \& Barrett, 2016), can be used to identify the role of experience in establishing these behaviors. Once this is understood, we can better predict when and why irrational choices are made and how to prevent them, if so desired.

\section{Considering the Role of Experience in the Formation of Biases}

A combined comparative, cross-cultural, and developmental approach has been extremely effective in other areas (c.f., Nielsen \& Haun, 2016). Consider the field of social learning. On the one hand, scientists have explored what young children across cultures choose to copy and in what circumstances. On the other hand, (many of the same) scientists have explored when other species, in particular non-human primates, learned socially. This work demonstrated that the roots of behaviors that were largely believed to be human-specific, such as cumulative culture (e.g., Tomasello, 1999; Whiten, McGuigan, Marshall-Pescini, \& Hopper, 2009), were present in other species. It also began to unpack the mechanism of social learning (i.e., imitation vs emulation; Gergely \& Csibra, 2006; Horner \& Whiten, 2005; Want \& Harris, 2002). In addition, these researchers demonstrated some of the limitations of other species' social learning, such as a general failure to consistently imitate precise motor movements (e.g., Tennie, Call, \& Tomasello, 2006, Want \& Harris, 2002), which suggested reasons why culture has made a stronger impact in humans than other species (Richerson \& Boyd, 2005). Overall, bringing together research from these populations has provided a rich basis for understanding the origins and development of humans' cultural abilities.

Within the context of decision making, this approach has great potential for addressing the basis of irrational tendencies. Consider the context: Organisms are faced with the difficult task of surviving and reproducing in a world in which decisions must be made quickly, with limited computational power and incomplete knowledge of alternatives. How does being continually confronted with these choices and their consequences impact later behavior? How does this experience shape later decision making?

Assuming that an individual's experiences do, in fact, impact decision making (an assumption that we will revisit below), developmental data should reflect this change. As children gain economic experience, perhaps first through exchanges with friends and family and later by entering more formal markets, they will begin to make choices that result in gains and losses. Further, some individuals may receive specific training or life experiences (e.g., careers, formal economic education). Measuring biases before and after these experiences can provide evidence of their impact. A related approach comes from cross-cultural work. Depending on the culture, there is variability in the degree to which individuals are involved in economic systems and decision making, thus cultural differences can also reveal the impact of experience on irrational decision making (Henrich et al., 2001).

Once there is evidence that experience plays a role in irrational tendencies, an important followup is to determine what that role is. Does experience limit irrational tendencies, or does it cause them? Intuitively, the former makes much more sense; experience should lead individuals to make better decisions, and it is hard to see value in learning patterns that make an actor less rational. Indeed, traditional economic theories predict that increased experience with markets should lead to a reduction in both irrational biases and irrational overall decision making, with more rational decision making observed in cases where subjects have extensive experience (e.g., through their jobs; List, 2003, 2004) or training 
(e.g., taking economics classes; Wang, Malhotra, \& Murnighan, 2011). Interestingly, though, for the biases we will address in this paper, there is no evidence of a reduction across childhood (see also, Harbaugh, Krause, \& Vesterlund, 2001). Even though children tend to have increased opportunities to earn and spend money through childhood (Otto, 2013), these decision-making experiences do not appear to lead to more rational decision making.

Instead, there is some evidence that experience with making these decisions has the opposite effect - that it may lead to the formation of behavioral biases (e.g., Reyna \& Brainerd, 2011). Although the idea that decision-making experience leads to more irrational tendencies may seem unlikely on first consideration, there are compelling theories to explain this pattern. Continually confronting similar problems may lead individuals to rely on past patterns of behavior (i.e., rules of thumb) instead of considering the specifics of each problem. Thus, instead of making individuals more rational, the often fast-paced, uncertain nature of economic participation may lead to the development of biases and encourage their use.

As we will expand upon below, findings on the development of framing effects are in line with this interpretation. In brief, at about age 5 to 6 years, children have been shown to choose differently depending on whether their options represent a gain or a loss. This tendency to consider the framing is found more consistently as children get older. Thus, it is possible that the economic opportunities Western children acquire by kindergarten and through childhood are shaping their responses to risky situations. Additionally, cross-cultural work shows evidence of the emergence of behavioral biases in groups exposed to formal Western markets (Apicella, Azevedo, Christakis, \& Fowler, 2014).

Interestingly, though, a different developmental pattern is shown in the other two cases that we review (the endowment effect and inequity aversion). In contrast to framing effects, these tendencies emerge early in development and at least some aspects of each seem to be relatively stable across experience. Which brings us to the assumption we teased above: Does experience really matter? And if so, how? What is the mechanism by which experience impacts these behavioral patterns differently?

This is where an evolutionary perspective becomes important. As noted above, everyday pressures to make important decisions with incomplete information may lead to the adoption of shortcuts in decision making. In addition to affecting a single life-span, however, these pressures may also have led to adaptations that support "fast-and-frugal" heuristics (Gigerenzer \& Todd, 1999), cognitive shortcuts that, despite their frugality, lead to adaptive behavior for the environment in which they evolved at minimal cost. If so, we may see evidence of early emergence in development, but also evidence of the same behavioral patterns across species.

In the next three sections we review developmental, comparative, and cross-cultural findings regarding the three behavioral biases listed above (framing effects, the endowment effect, and inequity aversion). These three cases were chosen because, for each, there is a relatively substantial body of developmental, comparative, and cross-cultural work to start to uncover patterns in the data. We contrast the developmental pattern shown in framing effects to that of the other two biases, and we provide possible explanations for, and impacts of, these differences. We also bring in cross-cultural and comparative data to provide a richer view of the role of experience and the evolutionary basis of these behavioral biases. In the final section, we address important points that come from this analysis of the data, including how to best conceptualize irrationalities, what may underlie their emergence, and how these behavioral patterns might be changed.

\section{Framing Effects}

A classic example of irrational decision making is framing effects, which describe the consistent finding that individuals vary their responses to risky situations depending on how the possible choices are described. Typically, subjects are more likely to choose a risky option when the consequences are framed in terms of losses as opposed to gains. Importantly, this is true even though the overall expected value is identical and only the way that the information presented is different. This finding has serious real-world implications. Just one example is a study showing that credit card customers made twice as many charges 
when benefits from using their cards were explained in terms of potential losses versus potential gains relative to using cash or checks (Ganzach \& Karsahi, 1995).

Framing effects seem to emerge relatively late during development, at least in comparison with the other two irrational tendencies we will discuss. Clear evidence for standard framing effects has been observed in adolescents (Chien, Lin, \& Worthley, 1996; Reyna et al., 2011), with a preference for risky choices to avoid losses growing stronger during childhood (Weller, Levin, \& Denburg, 2011). To date, the youngest age at which framing effects have been demonstrated is about 5 to 6 years (Levin \& Hart, 2003; Schlottmann \& Tring, 2005). Levin and Hart allowed children to select one of two boxes to add (gain frame) or subtract (loss frame) toys from a supply of toys that they would keep. One of the boxes was risky; it resulted in an equal chance of a change of either 0 or 2 toys to their supply. In contrast, the riskless box always resulted in a change of 1 toy. When losing toys, 5- to 6-year-olds were more likely to choose the risky box compared to when they were gaining toys.

This developmental pattern has been used to support a theory that decision-making experience may lead to biases. One mechanism by which this may happen is described by fuzzy-trace theory (e.g., Brainerd \& Reyna, 1990). By this account, when individuals initially consider a problem, they consider the specifics carefully and try to reason it out rationally. Thus, young children who have little experience with such choices initially consider the specific details of each problem and reason about them rationally to the best of their (e.g., mathematical) abilities (Reyna \& Ellis, 1994; c.f. Reyna \& Brainard, 2011). However, with increasing experience, individuals consider less about the specifics of the problem and rely more on the gist of it - a simplified (though possibly flawed) representation of the situation that is informed by their past experiences with similar problems. For framing effects, Reyna and Brainard propose that this gist may be like considering a problem as a dichotomy; e.g., gain frame: getting some vs chance of getting none; loss frame: losing some vs chance of not losing any. Such basic heuristics can simplify the reasoning needed. But, in doing so, they may lead to tendencies to make decisions that do not lead to optimal outcomes. By this account, individuals' decisions can become more based on shortcuts as compared to thoughtful, rational choices.

Because framing effects and other decision-making biases are irrational, in the sense that people's preferences do not consistently align, they are typically considered to be detrimental to the decision maker, on the assumption that choices influenced by these behavioral biases do not lead to the most optimal economic outcomes. However, fuzzy trace theory and other related theories propose that experience is actually creating these biases because they are, more often than not, beneficial. Although they may not lead to optimal economic outcomes in every circumstance, they simplify decision making, freeing up mental resources for other important tasks and increasing the odds of at least some decision being made, and, on average, they lead to the most beneficial behavior. Indeed, many biases are detrimental in only specific, often contrived, scenarios. The proposal that behavioral biases may be adaptive and useful challenges us to carefully consider their benefits and drawbacks, particularly when deciding whether we should change these patterns of behavior in the name of promoting more rational decision making.

Humans are not alone in these biases. Patterns of choices in loss frames relative to gain frames have been demonstrated in nonhuman primates (Chen, Lakshminarayanan, \& Santos, 2006; Lakshminarayanan, Chen, \& Santos, 2011) and even starlings (Marsh \& Kacelnik, 2002). In one procedure (Lakshminarayanan et al., 2011), capuchin monkeys were taught to exchange tokens with one of two experimenters in order to obtain food. One of the experimenters presented a riskless option; he or she gave the monkey two pieces of food on all trials. Trading with the other experimenter involved risk; he or she provided either one or three pieces of food (with an average payoff of two pieces). For half of the trials, the monkeys were first shown one piece of food by each experimenter. This served as a gain frame, as animals would always receive at least the number of pieces displayed. For the other half of the trials, the monkeys were initially shown three pieces of food, meaning that most choices resulted in a loss relative to this display. When each experimenter initially showed a single piece of food, monkeys were more likely to take the riskless option and obtain a sure two pieces of food. In contrast, when three pieces 
of food were initially shown, monkeys showed a preference for the risky option. Thus, like adult humans, the monkeys were more likely to choose a risky option in order to avoid a loss.

Considering the results from the developmental and comparative literature together presents an interesting picture: nonhuman primates and adults show evidence of framing effects, but young children often do not. One possible explanation for this pattern is that experience with decision making in markets (adults) or previous experiments (non-human primates) affects the development of these biases. The animals tested in these procedures are adults, and moreover, have typically participated in many decisionmaking experiments, which may have "formalized" their understanding of decision making in ways similar to what modern economic markets do to humans (see Apicella et al., 2014; Henrich et al., 2001). Thus, life experience with the gains and losses that accompany obtaining goods (such as food) may lead these species to develop a tendency to respond differently to risk depending on whether they are deciding about a gain or a loss. If this is the case, experience may have a negative effect on decision making, as these biases are emerging as children (or non-human primates) gain more experience with formal economic systems.

However, caution is warranted regarding our current understanding of the development of framing effects. Researchers have taken many steps to accommodate young children in their tasks. Considerations include targeting ages at which requisite concepts (e.g., expected value) are understood (e.g., Schlottmann \& Tring, 2005), adapting procedures to display the actual rewards of different outcomes (Reyna \& Ellis, 1994; Schlottmann \& Tring, 2005), and using only small numbers of rewards (Levin \& Hart, 2003). Even on these adapted tasks, young children's performance is inconsistent across different tests, with some procedures eliciting framing effects around age 5 (Levin \& Hart, 2003; Schlottmann \& Tring, 2005), and others failing to elicit them until later in childhood (e.g., Reyna \& Ellis, 1994; Weller et al., 2011). Further, the results of such studies are often complicated by children showing a general preference for the risky versus the riskless option (e.g., Harbaugh, Krause, \& Vesterlund, 2002; Levin \& Hart, 2003; Reyna \& Ellis, 1994), which reflects a common trend across a variety of behaviors in childhood (for review, see Boyer, 2006). Finally, studies with primates rarely involve actual losses (they may involve losing a potential reward, or getting nothing, but not taking away a previously-earned reward) because studies are done with foods that the primates eat immediately upon receipt. It may be that the lack of a true loss results in the primates experiencing the task very differently than the children do. As a result, we think it is premature to make firm conclusions about risk preferences in young children in the context of losses vs gain in comparison to non-human primates and adult humans, and encourage work that more directly replicates the procedures used with adult humans and, in particular, non-human primates. In most cases procedures developed for non-human primates easily adapt to work with children because they are simple, intuitive, and require no verbal instruction or, often, extensive pre-training.

In addition, further studies of the development of framing effects in other species would be useful for better understanding how decision-making experience may contribute to the emergence of these biases. In particular, it would be useful to track (or control) the experience animals have with exchanges involving risk. This is either challenging (i.e., requires multiple test sessions) or impossible (i.e., requires strict control of previous experience) in humans, so non-human species make an ideal model to develop hypotheses for further human testing.

\section{The Endowment Effect}

Related to framing effects is loss aversion, the tendency for individuals to prefer avoiding losses over making equivalent gains. One common manifestation of loss aversion is the Endowment Effect (EE). This phenomenon, which is well established in adults, is the tendency for individuals to value an object more upon owning it than they did previously (Kahneman, Knetsch, \& Thaler, 1991). For example, undergraduates wanted more money to sell a Final Four basketball ticket that they won in a raffle than they were otherwise willing to pay for the ticket (e.g., \$2400 to sell vs $\$ 160$ to buy; Carmon \& Ariely, 2000). 
In another classic demonstration of this effect (Knetsch, 1989), undergraduates were asked to choose which of two objects (a coffee mug or a chocolate bar) that they would like to keep. When allowed to freely choose between them, participants were equally likely to prefer the mug or the chocolate, resulting in a roughly equal number of choices of each across the group. However, in a manipulation where they were given one of the objects (instead of being allowed to freely choose between them) that they could then trade for the other, participants (as a group) showed a different pattern of preferences. As a group, these students showed a tendency to keep whichever object they were given, such that, on the whole, the group's preference shifted, presumably due only to the fact of possession. The EE has important practical significance for large-scale economic systems, as it can impede the efficient exchange of goods and property. It has also been implicated in problematic behaviors at the individual level (e.g., unhealthy food choices; Cramer \& Antonides, 2011).

Knetsch's (1989) trading paradigm is well suited for studies with populations that have limited language and cognitive abilities. Once the choice of either keeping the endowed object or trading for the alternative is established (through language for children, or through training to trade items for nonhuman primates), participants are faced with a decision that has meaningful first-person consequences. In tests of nonhuman primates, as in Knetsch's study, animals are endowed with one food item or object and then offered the chance to trade for a presumably equally attractive alternative. The tendency to keep an endowed food reward has been observed across the great apes and in capuchin monkeys, in both withinand between-subjects contexts (Brosnan, Jones, Gardner, Lambeth, \& Schapiro, 2012; Brosnan et al., 2007; Drayton, Brosnan, Carrigan, \& Stoinski, 2013; Flemming, Jones, Mayo, Stoinski, \& Brosnan, 2012; Kanngiesser, Santos, Hood, \& Call, 2011; Lakshminaryanan, Chen, \& Santos, 2008; results differ for non-food objects, which we discuss below). Most studies on non-human primates have found an endowment effect of roughly similar magnitude as is seen in human studies at the population level. Unlike human studies, most primate studies also report individual data, which indicate some variability among subjects. For instance, using an identical protocol across species, Brosnan and colleagues found that approximately $40 \%$ of a population of chimpanzees showed behavior consistent with an endowment effect ( $n=14$ of 33 subjects), whereas all of the gorillas tested did so $(n=9 ; 100 \%$; Brosnan et al., 2007; Drayton et al., 2013).

Similarly, past work indicates that children have a tendency to keep an endowed object rather than trading for an equally attractive alternative (Da Silva, Moreira, \& Da Costa Jr, 2014; Gelman, Manczak, \& Noles, 2012; Harbaugh et al., 2001; Lucas, Wagner, \& Chow, 2008). The youngest age at which this tendency has been observed using this trading methodology is 4 to 5 years. However, adaptations of this procedure reveal that children as young as 2 years show more subtle evidence of the endowment effect, with increased preferences for objects with which they have been endowed (Gelman et al., 2012). Indeed, the one study that has explicitly addressed the development of this bias found no significant differences between $3^{\text {rd }}$ graders, $5^{\text {th }}$ graders and adults (Harbaugh et al., 2001), indicating that the effect may emerge relatively early in development. However, it is important to note that work with 4year-olds has shown that only about $30 \%$ of children showed behavior consistent with an endowment effect (Lucas et al., 2008), so not all children are showing this effect so early. The proportion of adults who consistently show the EE is frequently not reported in studies. This information would be helpful for making clearer comparisons with children and nonhuman primates.

Overall, the data collected with children indicate that the EE emerges early during the lifespan and is relatively refractory to experience. This pattern fits well with comparative evidence showing that the EE is broadly present in nonhuman primates (both across species and individuals). Together, these findings suggest that this bias is not the result of activities specific to the complex culture or cognition characteristic of adult humans. Additionally, the fact that the EE seems to be relatively stable through childhood and into adulthood suggests that the bias is not substantively changed with increased engagement in everyday economic exchanges ${ }^{1}$. Contrary to formal economic theory, more economic decision-making experience does not appear to lead to more rational reasoning for children. Instead, as

\footnotetext{
${ }^{1}$ See Apicella et al., 2014, discussed below, for an exception to this.
} 
children grow older and make more economic decisions, they continue to show a tendency to overvalue keeping resources that they own, instead of focusing on which would be the best resource to have.

Positing an evolutionary basis for the endowment effect sheds light on possible factors that may influence when this pattern of behavior will be observed. For example, there is evidence that the endowment effect is stronger in nonhumans in situations that may be more relevant to survival. Although the endowment effect is often observed with foods, nonhuman primates generally do not show it for objects, such as toys (Brosnan et al., 2007, 2012; Kanngiesser et al., 2011). Indeed, this makes sense; exchanging one item for another item risks losing both (especially without formalized mechanisms to correct wrongs inflicted by a partner). Thus, it often may not be worth risking losing both for a slightly more preferred item. However, there is evidence that the endowment effect is more likely to be observed when a non-food object is a tool that can immediately be used to obtain food, versus when the same tool is presented outside of the immediate context of food acquisition, even when the food is visible (Brosnan et al., 2012; although see Kanngiesser et al., 2011). This suggests that animals have evolved to show endowment effects only for very salient objects, such as food or things that can immediately be used to get food. Finding such as these may help clear up the substantial unexplained variability in humans' expression of the endowment effect (Jones \& Brosnan, 2008).

Although we suspect that there are strong evolutionary underpinnings to the effect, recent findings from cross-cultural work with different human groups suggests that the relevance of economic exchange for a community may also impact the prevalence of the EE. Apicella and colleagues (2014) worked with the Hadza Bushmen in Tanzania. Traditionally, these individuals form a hunter-gatherer society that is characterized by communal sharing of resources and few market interactions. Individuals who live in very isolated Hadza communities maintain these traditional economies and also do not display an EE. In contrast, members of Hadza communities who interact with tourism groups and participate in more modern economic exchanges are more likely to display this bias. This can be interpreted as an influence of culture on the response, but one caveat to these results is that it is difficult to tell whether absence in a single instance is a result of psychological differences, or rather differences in how the population interpreted the situation or the game (Baumard \& Sperber, 2010).

Overall, the data indicate that the EE has a robust evolutionary history, appearing in similar patterns across many species of primates, suggesting a common underlying cause or mechanism. There is substantial variability in responses, with primate data suggesting that the effect is influenced by the evolutionary salience of the objects involved. Although there is some cultural variability in humans, it appears early in childhood and does not appear to change much during development, suggesting that it is relatively refractory to experience. Unfortunately, to our knowledge, there have been no developmental studies in primates, which are needed to test the hypothesis that the variability is influenced by evolutionary salience. Nonetheless, even though more work is needed to address these critical questions, results to date indicate that an evolutionary perspective can provide a useful framework for understanding the endowment effect.

\section{Inequity Aversion}

Although traditional economic theory holds that people's primary motivation is to increase their own outcomes, without regard for others', research over the past half century indicates that people have preferences about others' outcomes in addition to or in relation to their own (Güth \& Kocher, 2014). One such "social bias", inequity aversion, refers to individuals' tendency to dislike receiving less than (disadvantageous inequity aversion), or, in some cases, more than (advantageous inequity aversion), others receive (Loewenstein, Thompson, \& Bazerman, 1989; Shaw \& Olson, 2012). Inequity aversion can be economically irrational in both human and nonhuman primates, because it can lead subjects to sacrifice resources to obtain an equitable outcome, or even to protest inequity in a way that increases the shortterm discrepancy between themselves and their partner (i.e., spending energy and time, not to mention lost wages, protesting gaps in corporate wages, which does not typically immediately raise the protester's 
wages or decrease the higher wages). This results in absolute losses and, on occasion, even relative ones, if the protest does not change the other's outcome.

Looking-time measures provide evidence that even infants have some sensitivity to inequity. In one study, 21-month-old infants saw scenes in which two puppets received an equal number of stickers after completing varying amounts of work. Infants looked longer at these scenes when they had watched only one recipient clean up than when both puppets contributed equally (Sloane, Baillargeon, \& Premack, 2012). Infants also show a preference for fair distributors and are more likely to reach for a picture of an agent that had previously made fair distributions (Geraci \& Surian, 2011). In line with these findings, similar scenarios with adults elicit explicit negative reactions to inequity. For example, adults who saw videos of an actor rewarding two confederates inequitably (i.e., by giving one confederate a defective reward) were more likely to avoid that actor than were people who saw the actor provide the defective award to a single actor (thereby creating a comparable outcome with no inequity; Donovan \& Kelemen, 2011). These results suggest that an expectation for a correspondence between work and reward emerges from an early age.

Children also show active rejection of inequity at fairly young ages. For example, LoBue and colleagues gave pairs of preschool children varying numbers of stickers for cleaning up. Children who received fewer stickers than their partners did not refuse their stickers, but demonstrated inequity aversion through emotional and verbal reactions to their outcomes (LoBue, Nishida, Chiong, DeLoache, \& Haidt, 2011). In a simplified version of the ultimatum game, McAuliffe and colleagues (2013) presented 4- to 9year-old children with an "inequality game" in which children could accept or reject varying distributions of candies. Children at all ages rejected unequal distributions, even when they were playing alone, but they were more likely to reject unequal distributions when another child received a greater reward (McAuliffe, Blake, Kim, Wrangham, \& Warneken, 2013). However, it is worth noting that such patterns have not been observed in children in all cultures. In Uganda, where individuals typically have few resources and the culture emphasizes cooperation, 6- and 7-year-olds were more likely to give an unequal distribution to two peers, rather than discard resources (Paulus, 2015), indicating that background and experience may affect behaviors regarding inequity by these ages.

By age eight, children sometimes go so far as to reject even advantageous offers (Blake \& McAuliffe, 2011), although such rejections are substantially rarer than rejections of disadvantageous offers and do not manifest at all in more than half of seven cultures tested in a recent study (Blake et al., 2015). In general, however, as children in Western cultures get older they show evidence of inequity aversion on a wider range of tasks, many of which can be very demanding in terms of cognitive skills or executive functioning.

Another common test of inequity aversion is the ultimatum game (UG), a task in which a proposer offers some division of a quantity of resources, which a responder can either accept to get their share, or reject in which case neither player gets anything. Children tend to accept (e.g., Benenson, Pascoe, \& Radmore, 2007; Harbaugh, Krause, \& Liday, 2003; Melis, Floedl, \& Tomasello, 2015) and propose (e.g., Benenson et al., 2007; Wittig, Jensen, \& Tomasello, 2013, though see also Murnighan \& Saxon, 1998) significantly lower offers (more rational) than do Western adults (see Henrich, 2000 for a report of cross-cultural differences). Overall, however, given its linguistic and mathematical demands, coupled with the relatively brief training typically provided, the UG may be a particularly difficult task for young children (e.g., Murnighan \& Saxon, 1998), even in a modified format used with other species (Proctor, Williamson, de Waal, \& Brosnan, 2013). Additionally, researchers cannot agree on what adult refusals mean on this task. Are subjects driven by reciprocity, punishment, spite, or a sense of fairness (Camerer, 2003), and if the latter, what do we even mean by fairness (Debove, Baumard, \& André, 2016)? Although performance on the UG suggests different behavior in young children than adults, a broad perspective on the literature suggests a developmental pattern similar to that seen in the endowment effect; children show a basic tendency to be averse to inequity early in development that increases as children become better able to understand complex exchanges and to control their impulses.

Comparative findings also reveal a tendency towards inequity aversion across a wide range of non-human primate species. There has been work on the ultimatum game in chimpanzees, although the 
task cannot be used in its original format due to linguistic demands, making comparisons challenging. Instead, experimenters have used a modified "limited form" task, in which proposers must choose which of two distributions and responders can either accept by continuing the task (i.e., returning a token or pulling in a tray of food) or refuse by doing nothing (i.e., passively refuse). There is no evidence that participants ever refuse any offer (Jensen, Call, \& Tomasello, 2007; Kaiser, Jensen, Call, \& Tomasello, 2012; Proctor et al., 2013), although, in one study, subjects were more likely to choose the equal distribution when their partner had recourse to refuse than when they did not, suggesting that the proposers were sensitive to their partner's actions (Proctor et al., 2013). Moreover, children also do not refuse in this modified task (Proctor et al., 2013), and other evidence suggests that doing nothing is hard for even adults, suggesting that it is not a good "choice" (Smith \& Silberberg, 2010) and perhaps explaining the low level of refusals in these tasks. Even aside from these challenges, the ultimatum game likely requires a great number of cognitive skills, suggesting that simpler tasks are more appropriate for other species and, perhaps, children.

One related task is the Impunity game, in which subjects have the option to accept or refuse offers but it does not impact their partner's outcome. Little work has been done on this task due to the assumption that no rational individual would ever refuse in this case, as it increases relative inequity. However, research suggests that people routinely refuse unequal offers despite this (in the UG, a refusal means that neither participant gets the reward, thereby decreasing absolute outcomes but making them relatively equal; Lamichhane, Adhikari, Brosnan, \& Dhamala, 2014; Yamagishi et al., 2009). Quite a lot of work on other species has used a conceptually similar protocol exploring whether subjects refuse rewards when their partner gets a better one, but they cannot influence the partner's outcome.

In these tasks, pairs of monkeys are given tokens that they can trade back to the experimenter for food rewards (Brosnan \& de Waal, 2003; reviewed in Brosnan, 2013 Brosnan \& de Waal, 2014). The researchers vary the value of rewards given to each subject. In the critical manipulation, the target animal receives a lower-value reward (e.g., a cucumber) after watching its partner receive a higher-value reward (e.g., a grape). Monkeys that received a less preferred outcome than a partner were less likely to exchange tokens with the experimenter and/or accept rewards than were monkeys in conditions in which all animals received the same (less preferred) rewards for the task. Thus, the animals' decisions to participate suggest that they considered what rewards others received as well as their own. Other studies with other nonhuman species have reported similar results (reviewed in Brosnan \& de Waal, 2014; McGetrick \& Range, 2018; see also Oberliessen et al., 2016; Wascher \& Bugnyar, 2013); this consistent pattern of results across young children and a number of primate species indicates that there may be an evolutionarily-based tendency toward inequity aversion shared by many, although not all, primate species.

Again, similar to work on the endowment effect, this cross-species work is uncovering potential evolutionary pressures that may drive the emergence of irrational tendencies. In particular, only some species respond to inequity, and this response seems to be related to whether the situation is social (Leimgruber, Rosati, \& Santos, 2016) and the degree to which they cooperate with non-kin (for a review, see Brosnan, 2013; Brosnan \& de Waal, 2014. See also a special double issue of Social Justice Research, Volume 25, Issues 2 and 3, 2012). Species that cooperate routinely, including capuchin monkeys, chimpanzees, macaques, dogs, wolves, rats, and some corvids, show responses to inequity in these experimental tasks (Brosnan \& de Waal, 2014 and see above references). Those that do not, including orangutans, squirrel monkeys, and other corvids, do not respond, nor do cooperative breeders (marmosets, tamarins, and owl monkeys), for whom the cost of finding a new social partner may be too high to merit a negative reaction for such a small inequality. Based on the current evidence, this distribution cannot be explained by homology, social group size or structure, or brain size, so although future work may uncover additional factors that are influencing responses, they are currently best explained as a response that cooccurs with cooperation (Brosnan \& de Waal, 2014).

Additional work supports this. In several studies, exploring how inequity impacts cooperation, results suggest that primates are focused on their partner's behavior. For instance, chimpanzees prefer to cooperate with partners who tolerantly shared food with them in other contexts (Melis, Hare, \& 
Tomasello, 2006a, b). Capuchin monkeys are more likely to cooperate when rewards are not monopolizeable (de Waal \& Davis, 2003) and when partners do not take all the advantages (Brosnan, Freeman, \& de Waal, 2006). In the latter study, capuchins cooperated even for unequal distributions as long as both partners sometimes got the better option, but ceased cooperating when one partner began monopolizing the preferred outcome. These results suggest that we may be happy to tolerate inequality as long as our partners play fair, or if rewards "even out" over the long run. This is good news, as there are few situations in which outcomes are consistently identical across partners, so an inability to take the larger context into account would stifle cooperation.

There is still debate as to whether this is the appropriate characterization of what drives inequity aversion, with some theorists arguing that a more general, nonsocial mechanism underlies disadvantageous inequity aversion (Chen \& Santos, 2006; McAuliffe et al., 2013). This proposal is supported by developmental research that shows that the social context becomes increasingly important in children's inequity aversion as they get older (Bereby-Meyer \& Fiks, 2013; McAuliffe et al., 2013). We desperately need more developmental data from a variety of different cultures and contexts to help identify which features are highly conserved, and are therefore likely to be more tightly tied to biology, and which features may require specific experiences or contexts to develop. In addition to the crosscultural data, work with species that did not evolve to live in complex social groups might help to resolve this issue by clarifying which behaviors require a social context to evolve (if they do not appear in relatively less social species) or develop (if they appear in both more and less social species, but only with social exposure, such as group housing). Overall, a better understanding of these reactions could lead to more efficient strategies for accurately assessing and appropriately reacting to inequalities.

Again, the comparative data support the need to understand context. In no study of chimpanzees has every subject responded to inequity, and in a few cases, there has been no response at all (Bräuer, Call, \& Tomasello, 2006, 2009; Brosnan et al., 2015; Brosnan, Schiff, \& de Waal, 2005; Brosnan, Talbot, Ahlgren, Lambeth, \& Schapiro, 2010; Engelmann, Clift, Herrmann, \& Tomasello, 2017). Similarly, responses in capuchin monkeys are highly variable across studies (Brosnan \& de Waal, 2003; Brosnan, Houser, et al., 2010; Dubreuil, Gentile, \& Visalberghi, 2006; Fletcher, 2008; Fontenot, Watson, Roberts, \& Miller, 2007; McAuliffe et al., 2015; Silberberg, Crescimbene, Addessi, Anderson, \& Visalberghi, 2009; Takimoto, Kuroshima, \& Fujita, 2009; Talbot et al., 2018; van Wolkenten, Brosnan, \& de Waal, 2007). What does this mean? A sufficient number of studies in different labs have found an effect that it is unlikely that it is just a particularly fragile reaction. Therefore, it seems likely that context is driving the subjects' reactions. To date, although a number of factors have been proposed, there is not one that explains all of the variation, although it seems likely that it is a combination of procedural effects (i.e., the value of the rewards; Talbot et al., 2018, or the orientation of the subjects to one another; Brosnan et al., 2015) and personal factors (rank, sex, age, personality and experience). Understanding this in other species, for which we have more ability to control context, will provide hypotheses to test in humans as well, and clarify both why this response evolved and what role experience plays in guiding reactions.

\section{Conclusions}

Considering developmental, comparative, and cross-cultural work on irrational behavior provides a much broader foundation for understanding the role of experience in shaping decisions and, in particular, allows us to directly explore two different ways that decision-making experience may lead to the formation of behavioral biases; one within an individual's lifespan and one on an evolutionary time frame. Current data indicate that both ontological and phylogenetic influences impact different behavioral biases.

Research on framing effects suggests that some behavioral biases may be the result of individuals gaining experience making decisions; organisms may come to rely on the outcomes of their past behaviors, or heuristics, to make decisions about novel situations, particularly when decisions are complicated, cognitive resources are already taxed, or they have incomplete information. Being able to fall back on rules of thumb that, on average, result in beneficial outcomes simplifies decision making 
while leading to outcomes that are, on average, good enough. Although there are occasionally costs due to sub-optimal payoffs, the benefits of simplified decision making likely make this a worthwhile tradeoff.

Studies of both the endowment effect and inequity aversion, on the other hand, suggest a stronger impact of evolutionary history. Both of these biases are present early in development and persist throughout childhood. Further, nonhuman species exhibit them in at least some contexts. Together, this pattern of findings emphasizes the role of biology. However, one challenge is disentangling the everyday experiences of young (Western) children and lab animals from biology; although we see a different pattern in these biases than in framing effects (largely using the same Western and lab populations), it is possible that the experiential effects of the endowment effect and inequity aversion come from even earlier experiences, before the age at which these tests are typically run, which would make them challenging to find. To determine this, we need studies that carefully track the economic opportunities of very young children or naive animals to determine their trajectory of decision making. Of course, even if experience is involved to some degree, the developmental and comparative results indicate that there is a biological basis to these biases.

An important benefit of taking an evolutionary perspective is that it sheds light on the question of what features of our ancestors' environments may have selected for these behavioral patterns (Gigerenzer $\&$ Todd, 1999). For example, the endowment effect may have evolved as a solution to the problem of risky exchange (Jones \& Brosnan, 2008). Because exchange carries the risk of losing an object or food item without receiving anything in return, animals that hold onto a valuable object rather than risk it in trade may find themselves at an advantage over the long term, especially when the objects in question are relevant to survival and there are no formalized mechanisms to remonstrate. Inequity aversion, as a heuristic, allows an individual to identify and reject partners with whom cooperation routinely yields unequal outcomes. Because evolution is a game of relative payoffs, the absolute utility of a given outcome matters less than the advantage it provides an actor relative to other individuals, making a refusal that results in relative equity the better outcome, even if it means an absolute loss in resources (Brosnan, 2013). For this reason, an individual may do best if it can quickly assess how valuable the current relationship is and respond in a way that avoids disadvantageous partnerships. Of course, the findings discussed in this review are based on results that are found in laboratory settings, which are not the same as either ancestral or modern environments. However, the ability to control variables makes these studies a valuable contribution for understanding the impact of specific contexts and the evolutionary trajectory that they may have followed.

One practical implication for considering the role of experience in the manifestation of biases is that it can help determine the best practices for limiting the maladaptive application of irrational tendencies. If a bias is evolutionarily-based and/or early developing, it may be deeply ingrained and relatively resistant to formal intervention. If so, explicit, top-down strategies may be the best way to overcome biases that manifest in problematic ways. Individuals could be trained to recognize common errors and given strategies for overcoming them. Given the metacognition and awareness that are necessary for such interventions to work, adults and adolescents are the proper targets of such interventions. In contrast, a prolonged development and a role for experience opens the possibility that a bias may be more malleable and subject to systemized training. Experience with the optimal solutions to problems could be provided, for example, through game-like tasks throughout the lifespan to avoid the formation of these biases.

In sum, in addition to providing a better characterization of thinking throughout the lifespan, a combined developmental, cross-cultural, and comparative perspective on decision making has important theoretical and practical implications. As illustrated by the three biases reviewed here, considering the role of experience can enhance our understanding of why decision making may not conform to traditional definitions of economic rationality and can help highlight possible benefits to these biases. Finally, pinpointing the role of experience can help determine the malleability of decision making. The expanded use of this approach would allow for more systematic comparisons across biases and would help resolve the degree to which key experiences are responsible for irrational decision-making tendencies. 


\section{Acknowledgements}

This work was supported by the National Science Foundation SES 1357605 to RW and SFB.

\section{References}

Apicella, C. L., Azevedo, E. M., Christakis, N. A., \& Fowler, J. H. (2014). Evolutionary origins of the endowment effect: Evidence from hunter-gatherers. American Economic Review, 104, 1793-1805.

Apicella, C. L., \& Barrett, H. C. (2016). Cross-cultural evolutionary psychology. Current Opinion in Psychology, 7, 9297.

Baumard, N., \& Sperber, D. (2010). Weird people, yes, but also weird experiments. Behavioral and Brain Sciences, 33, 84-85.

Becker, G. S. (1976). The economic approach to human behavior. Chicago: University of Chicago Press.

Benenson, J. F., Pascoe, J., \& Radmore, N. (2007). Children's altruistic behavior in the dictator game. Evolution and Human Behavior, 28, 168-175.

Bereby-Meyer, Y., \& Fiks, S. (2013). Changes in negative reciprocity as a function of age. Journal of Behavioral Decision Making, 26, 397-403.

Blake, P. R., \& McAuliffe, K. (2011). "I had so much it didn't seem fair": Eight-year-olds reject two forms of inequity. Cognition, 120, 215-224.

Blake, P. R., McAuliffe, K., Corbit, J., Callaghan, T. C., Barry, O., Bowie, A., ...Warneken, F. (2015). The ontogeny of fairness in seven societies. Nature, 528, 258-261.

Boyer, T. W. (2006). The development of risk-taking: A multi-perspective review. Developmental Review, 26, 291345.

Brainerd, C. J., \& Reyna, V. F. (1990). Gist is the grist: Fuzzy-trace theory and the new intuitionism. Developmental Review, 10, 3-47.

Bräuer, J., Call, J., \& Tomasello, M. (2006). Are apes really inequity averse? Proceedings of the Royal Society B: Biological Sciences, 273, 3123-3128.

Bräuer, J., Call, J., \& Tomasello, M. (2009). Are apes inequity averse? New data on the token-exchange paradigm. American Journal of Primatology, 71, 175-181.

Brosnan, S. F. (2013). Justice-and fairness-related behaviors in nonhuman primates. Proceedings of the National Academy of Sciences, 110 (Supplement 2), 10416-10423.

Brosnan, S. F., \& de Waal, F. B. (2003). Monkeys reject unequal pay. Nature, 425, 297-299.

Brosnan, S. F., \& de Waal, F. B. (2014). Evolution of responses to (un) fairness. Science, 346, 1251776.

Brosnan, S. F., Freeman, C., \& de Waal, F. B. M. (2006). Partner's behavior, not reward distribution, determines success in an unequal cooperative task in capuchin monkeys. American Journal of Primatology, 68, 713724.

Brosnan, S. F., Hopper, L. M., Richey, S., Freeman, H. D., Talbot, C. F., Gosling, S. D., ...Schapiro, S. J. (2015). Personality influences responses to inequity and contrast in chimpanzees. Animal Behavior, 101, 75-87.

Brosnan, S. F., Houser, D., Leimgruber, K., Xiao, E., Chen, T., \& de Waal, F. B. M. (2010). Competing demands of prosociality and equity in monkeys. Evolution and Human Behavior, 31, 279-288.

Brosnan, S. F., Jones, O. D., Gardner, M., Lambeth, S. P., \& Schapiro, S. J. (2012). Evolution and the expression of biases: Situational value changes the endowment effect in chimpanzees. Evolution and Human Behavior, 33, 378-386.

Brosnan, S. F., Jones, O. D., Lambeth, S. P., Mareno, M. C., Richardson, A. S., \& Schapiro, S. J. (2007). Endowment effects in chimpanzees. Current Biology, 17, 1704-1707.

Brosnan, S. F., Schiff, H. C., \& de Waal, F. B. M. (2005). Tolerance for inequity may increase with social closeness in chimpanzees. Proceedings of the Royal Society B: Biological Sciences, 272, 253-258.

Brosnan, S. F., Talbot, C., Ahlgren, M., Lambeth, S. P., \& Schapiro, S. J. (2010). Mechanisms underlying responses to inequitable outcomes in chimpanzees, Pan troglodytes. Animal Behaviour, 79, 1229-1237.

Camerer, C. (2003). Behavioral game theory: Experiments in strategic interaction. Princeton, NJ: Princeton University Press.

Carmon, Z., \& Ariely, D. (2000). Focusing on the forgone: How value can appear so different to buyers and sellers. Journal of Consumer Research, 27, 360-370.

Chen, M. K., Lakshminarayanan, V., \& Santos, L. R. (2006). How basic are behavioral biases? Evidence from capuchin monkey trading behavior. Journal of Political Economy, 114, 517-537. 
Chen, M. K., \& Santos, L. R. (2006). Some thoughts on the adaptive function of inequity aversion: An alternative to Brosnan's social hypothesis. Social Justice Research, 19, 201-207.

Chien, Y.-C., Lin, C., \& Worthley, J. (1996). Effect of framing on adolescents' decision making. Perceptual and Motor Skills, 83, 811-819.

Cramer, L., \& Antonides, G. (2011). Endowment effects for hedonic and utilitarian food products. Food Quality and Preference, 22, 3-10.

Da Silva, S., Moreira, B., \& Da Costa Jr, N. (2014). Preschoolers and the endowment effect. PloS One, 9, e109520.

Debove, S., Baumard, N., \& Andre, J.-B. (2016). Models of the evolution of fairness in the ultimatum game: A review and classification. Evolution and Human Behavior, 37, 245-254.

de Waal, F., \& Davis, J. M. (2003). Capuchin cognitive ecology: Cooperation based on projected returns. Neuropsychologia, 41, 221-228.

Donovan, E., \& Kelemen, D. (2011). Just rewards: Children and adults equate accidental inequity with intentional unfairness. Journal of Cognition and Culture, 11, 137-150.

Drayton, L. A., Brosnan, S. F., Carrigan, J., \& Stoinski, T. S. (2013). Endowment effects in gorillas (Gorilla gorilla). Journal of Comparative Psychology, 127, 365-369.

Dubreuil, D., Gentile, M. S., \& Visalberghi, E. (2006). Are capuchin monkeys (Cebus apella) inequity averse? Proceedings of the Royal Society B: Biological Sciences, 273, 1223-1228.

Engelmann, J. M., Clift, J. B., Herrmann, E., \& Tomasello, M. (2017). Social disappointment explains chimpanzees' behaviour in the inequity aversion task. Proceedings of the Royal Society B: Biological Sciences, 284, 20171502.

Flemming, T. M., Jones, O. D., Mayo, L., Stoinski, T., \& Brosnan, S. F. (2012). The endowment effect in orangutans. International Journal of Comparative Psychology, 25, 285-298.

Fletcher, G. E. (2008). Attending to the outcome of others: Disadvantageous inequity aversion in male capuchin monkeys (Cebus apella). American Journal of Primatology, 70, 901-905.

Fontenot, M. B., Watson, S. L., Roberts, K. A., \& Miller, R. W. (2007). Effects of food preferences on token exchange and behavioural responses to inequality in tufted capuchin monkeys, Cebus apella. Animal Behavior, 74, 487-496.

Ganzach, Y., \& Karsahi, N. (1995). Message framing and buying behavior: A field experiment. Journal of Business Research, 32, 11-17.

Gelman, S. A., Manczak, E. M., \& Noles, N. S. (2012). The nonobvious basis of ownership: Preschool children trace the history and value of owned objects. Child Development, 83, 1732-1747.

Geraci, A., \& Surian, L. (2011). The developmental roots of fairness: Infants' reactions to equal and unequal distributions of resources. Developmental Science, 14, 1012-1020.

Gergely, G., \& Csibra, G. (2006). Sylvia's recipe: The role of imitation and pedagogy in the transmission of cultural knowledge. In N. J. Enfield \& S. C. Levenson (Eds), Roots of human sociality: Culture, cognition, and human interaction (p. 229-255). Oxford, UK: Berg Publishers.

Gigerenzer, G., \& Todd, P. M. (1999). Simple heuristics that make us smart. New York, NY, Oxford University Press, USA.

Güth, W., \& Kocher, M. G. (2014). More than thirty years of ultimatum bargaining experiments: Motives, variations, and a survey of the recent literature. Journal of Economic Behavior \& Organization, 108, 396409.

Harbaugh, W. T., Krause, K., \& Liday, S. J. (2003). Bargaining by children. University of Oregon Economics Working Papers, No. 2002-2004. Available at SSRN: https://ssrn.com/abstract=436504 or http://dx.doi.org/10.2139/ssrn.436504

Harbaugh, W. T., Krause, K., \& Vesterlund, L. (2001). Are adults better behaved than children? Age, experience, and the endowment effect. Economics Letters, 70, 175-181.

Harbaugh, W. T., Krause, K., \& Vesterlund, L. (2002). Risk attitudes of children and adults: Choices over small and large probability gains and losses. Experimental Economics, 5, 53-84.

Henrich, J. (2000). Does culture matter in economic behavior? Ultimatum game bargaining among the Machiguenga of the Peruvian Amazon. The American Economic Review, 90, 973-979.

Henrich, J., Boyd, R., Bowles, S., Camerer, C., Fehr, E., Gintis, H., \& McElreath, R. (2001). In search of Homo Economicus: Behavioral experiments in 15 small-scale societies. American Economic Review, 91, 73-78.

Horner, V., \& Whiten, A. (2005). Causal knowledge and imitation/emulation switching in chimpanzees (Pan troglodytes) and children (Homo sapiens). Animal Cognition, 8, 164-181.

Jensen, K., Call, J., \& Tomasello, M. (2007). Chimpanzees are rational maximizers in an ultimatum game. Science, 318, 107-109. 
Jones, O. D., \& Brosnan, S. F. (2008). Law, biology, and property: A new theory of the endowment effect, William and Mary Law Review, 49, 1935-1990.

Kahneman, D., Knetsch, J. L., \& Thaler, R. H. (2008). The endowment effect: Evidence of losses valued more than gains. Handbook of Experimental Economics Results, 1, 939-948.

Kaiser, I., Jensen, K., Call, J., \& Tomasello, M. (2012). Theft in an ultimatum game: Chimpanzees and bonobos are insensitive to unfairness. Biology Letters, 8, 942-945.

Kanngiesser, P., Santos, L. R., Hood, B. M., \& Call, J. (2011). The limits of endowment effects in great apes (Pan paniscus, Pan troglodytes, Gorilla gorilla, Pongo pygmaeus). Journal of Comparative Psychology, 125, 436-445.

Knetsch, J. L. (1989). The endowment effect and evidence of nonreversible indifference curves. The American Economic Review, 79, 1277-1284.

Lakshminaryanan, V., R., Chen, M., \& Santos, L. R. (2008). Endowment effect in capuchin monkeys. Philosophical Transactions of the Royal Society B: Biological Sciences, 363, 3837-3844.

Lakshminarayanan, V. R., Chen, M. K., \& Santos, L. R. (2011). The evolution of decision-making under risk: Framing effects in monkey risk preferences. Journal of Experimental Social Psychology, 47, 689-693.

Lamichhane, B., Adhikari, B. M., Brosnan, S. F., \& Dhamala, M. (2014). The neural basis of perceived unfairness in economic exchanges. Brain Connectivity, 4, 619-630.

Leimgruber, K. L., Rosati, A. G., \& Santos, L. R. (2016). Capuchin monkeys punish those who have more. Evolution and Human Behavior, 37, 236-244.

Levin, I. P., \& Hart, S. S. (2003). Risk preferences in young children: Early evidence of individual differences in reaction to potential gains and losses. Journal of Behavioral Decision Making, 16, 397-413.

List, J. A. (2003). Does market experience eliminate market anomalies? Quarterly Journal of Economics, 118, 4172.

List, J. A. (2004). Neoclassical theory versus prospect theory: Evidence from the marketplace. Econometrica, 72, 615-625.

LoBue, V., Nishida, T., Chiong, C., DeLoache, J. S., \& Haidt, J. (2011). When getting something good is bad: Even three-year-olds react to inequality. Social Development, 20, 154-170.

Loewenstein, G. F., Thompson, L., \& Bazerman, M. H. (1989). Social utility and decision making in interpersonal contexts. Journal of Personality and Social Psychology, 57, 426-441.

Lucas, M. M., Wagner, L., \& Chow, C. (2008). Fair game: The intuitive economics of resource exchange in fouryear olds. Journal of Social, Evolutionary, and Cultural Psychology, 2, 74-88.

Marsh, B., \& Kacelnik, A. (2002). Framing effects and risky decisions in starlings. Proceedings of the National Academy of Sciences, 99, 3352-3355.

McAuliffe, K., Blake, P. R., Kim, G., Wrangham, R. W., \& Warneken, F. (2013). Social influences on inequity aversion in children. PloS One, 8 , e80966.

McAuliffe, K., Chang, L. W., Leimgruber, K. L., Spaulding, R., Blake, P. R., \& Santos, L. R. (2015). Capuchin monkeys, Cebus apella, show no evidence for inequity aversion in a costly choice task. Animal Behaviour, $103,65-74$.

McGetrick, J., \& Range, F. (2018). Inequity aversion in dogs: A review. Learning \& Behavior, 4, 479-500.

Melis, A. P., Floedl, A., \& Tomasello, M. (2015). Non-egalitarian allocations among preschool peers in a face-toface bargaining task. PloS One, 10, e0120494-e0120494.

Melis, A. P., Hare, B., \& Tomasello, M. (2006a). Chimpanzees recruit the best collaborators. Science, 311, 12971300.

Melis, A. P., Hare, B., \& Tomasello, M. (2006b). Engineering cooperation in chimpanzees: Tolerance constraints on cooperation. Animal Behavior, 72, 275-286.

Murnighan, J. K., \& Saxon, M. S. (1998). Ultimatum bargaining by children and adults. Journal of Economic Psychology, 19, 415-445.

Nielsen, M., \& Haun, D. (2016). Why developmental psychology is incomplete without comparative and crosscultural perspectives. Philosophical Transactions of the Royal Society B: Biological Sciences, 371, 20150071.

Oberliessen, L., Hernandez-Lallement, J., Schäble, S., van Wingerden, M., Seinstra, M., \& Kalenscher, T. (2016). Inequity aversion in rats, Rattus norvegicus. Animal Behaviour, 115, 157-166.

Otto, A. (2013). Saving in childhood and adolescence: Insights from developmental psychology. Economics of Education Review, 33, 8-18.

Paulus, M. (2015). Children's inequity aversion depends on culture: A cross-cultural comparison. Journal of Experimental Child Psychology, 132, 240-246. 
Proctor, D., Williamson, R. A., de Waal, F. B., \& Brosnan, S. F. (2013). Chimpanzees play the ultimatum game. Proceedings of the National Academy of Sciences, 110, 2070-2075.

Reyna, V. F., \& Brainerd, C. J. (2011). Dual processes in decision making and developmental neuroscience: A fuzzy-trace model. Developmental Review, 31, 180-206.

Reyna, V. F., \& Ellis, S. C. (1994). Fuzzy-trace theory and framing effects in children's risky decision making. Psychological Science, 5, 275-279.

Reyna, V. F., Estrada, S. M., DeMarinis, J. A., Myers, R. M., Stanisz, J. M., \& Mills, B. A. (2011). Neurobiological and memory models of risky decision making in adolescents versus young adults. Journal of Experimental Psychology: Learning, Memory, and Cognition, 37, 1125-1142.

Richerson, P. J., \& Boyd, R. (2005). Not by genes alone: How culture transformed human evolution. Chicago: University of Chicago Press.

Schlottmann, A., \& Tring, J. (2005). How children reason about gains and losses: Framing effects in judgement and choice. Swiss Journal of Psychology, 64, 153-171.

Shaw, A., \& Olson, K. R. (2012). Children discard a resource to avoid inequity. Journal of Experimental Psychology: General, 141, 382-395.

Silberberg, A., Crescimbene, L., Addessi, E., Anderson, J. R., \& Visalberghi, E. (2009). Does inequity aversion depend on a frustration effect? A test with capuchin monkeys (Cebus apella). Animal Cognition, 12, 505509.

Sloane, S., Baillargeon, R., \& Premack, D. (2012). Do infants have a sense of fairness? Psychological Science, 23, 196-204.

Smith, P., \& Silberberg, A. (2010). Rational maximizing by humans (Homo sapiens) in an ultimatum game. Animal Cognition, 13, 671-677.

Takimoto, A., Kuroshima, H., \& Fujita, K. (2009). Capuchin monkeys (Cebus apella) are sensitive to others' reward: An experimental analysis of food-choice for conspecifics. Animal Cognition, 13, 249-261.

Talbot, C. F., Parrish, A. E., Watzek, J., Essler, J. L., Leverett, K. L., Paukner, A., \& Brosnan, S. F. (2018). The influence of reward quality and quantity and spatial proximity on the responses to inequity and contrast in capuchin monkeys (Cebus [Sapajus] apella). Journal of Comparative Psychology, 132, 75-87.

Tennie, C., Call, J., \& Tomasello, M. (2006). Push or pull: Imitation vs. emulation in great apes and human children. Ethology, 112, 1159-1169.

Tomasello, M. (1999). The cultural origins of human cognition. Cambridge, MA, Harvard University Press.

van Wolkenten, M., Brosnan, S. F., \& de Waal, F. B. (2007). Inequity responses of monkeys modified by effort. Proceedings of the National Academy of Sciences, 104, 18854-18859.

Wang, L., Malhotra, D., \& Murnighan, J. K. (2011). Economics education and greed. Academy of Management Learning \& Education, 10, 643-660.

Want, S. C., \& Harris, P. L. (2002). How do children ape? Applying concepts from the study of non-human primates to the developmental study of 'imitation' in children. Developmental Science, 5, 1-14.

Wascher, C. A. F., \& Bugnyar, T. (2013). Behavioral responses to inequity in reward distribution and working effort in crows and ravens. PLOS ONE, 8, e56885.

Weller, J. A., Levin, I. P., \& Denburg, N. L. (2011). Trajectory of risky decision making for potential gains and losses from ages 5 to 85. Journal of Behavioral Decision Making, 24, 331-344.

Whiten, A., McGuigan, N., Marshall-Pescini, S., \& Hopper, L. M. (2009). Emulation, imitation, over-imitation and the scope of culture for child and chimpanzee. Philosophical Transactions of the Royal Society B, 364, 2417-2428.

Wittig, M., Jensen, K., \& Tomasello, M. (2013). Five-year-olds understand fair as equal in a mini-ultimatum game. Journal of Experimental Child Psychology, 116, 324-337.

Yamagishi, T., Horita, Y., Takagishi, H., Shinada, M., Tanida, S., \& Cook, K. S. (2009). The private rejection of unfair offers and emotional commitment. Proceedings of the National Academy of Sciences, 106, 1152011523. 